\title{
Tidally induced radial-velocity variations in close binaries
}

\author{
B. Willems ${ }^{1}$ and C. Aerts ${ }^{2}$ \\ 1 Department of Physics and Astronomy, The Open University, Walton Hall, Milton Keynes, MK7 6AA, UK \\ e-mail: B.Willems@open.ac.uk \\ 2 Instituut voor Sterrenkunde, Katholieke Universiteit Leuven, Celestijnenlaan 200 B, 3001 Leuven, Belgium \\ e-mail: Conny. Aerts@ster.kuleuven.ac.be
}

Received 25 July 2001 / Accepted 4 January 2002

\begin{abstract}
A theoretical framework for the determination of tidally induced radial-velocity variations in a component of a close binary is presented. Both the free and the forced oscillations of the component are treated as linear, isentropic perturbations of a spherically symmetric star. Resonances between dynamic tides and free oscillation modes are taken into account by means of the formalism developed by Smeyers et al. (1998). The amplitude of the tidally induced radial-velocity variations seen by the observer depends on the orbital eccentricity and on the orbital inclination. The amplitude increases with increasing orbital eccentricity and is most sensitive to the value of the orbital inclination when $20^{\circ} \lesssim i \lesssim 70^{\circ}$. In the case of a $5 M_{\odot}$ ZAMS star with a $1.4 M_{\odot}$ compact companion, it is shown that resonant dynamic tides can lead to radial-velocity variations with amplitudes large enough to be detected in observations. The shape of the tidally induced radial-velocity curves varies from very irregular for orbital periods away from any resonances with free oscillation modes to sinusoidal for orbital periods close to a resonance with a free oscillation mode. Our investigation is concluded with an application to the slowly pulsating B star HD 177863 showing the possibility of resonant excitation of a high-order second-degree $g^{+}$-mode in this star.
\end{abstract}

Key words. binaries: close - stars: oscillations - methods: analytical - stars: individual: HD 177863

\section{Introduction}

In close binary systems of stars, each component is subject to the time-dependent tidal force exerted by its companion. A commonly used approach for the study of tidal effects in close binaries is based on the expansion of the tide-generating potential in Fourier series in terms of multiples of the companion's mean motion. Through these expansions, the tidal action of the companion induces an infinite number of partial dynamic tides in the star, each with its own forcing angular frequency.

In binaries with shorter orbital periods, the forcing angular frequencies may be close to the eigenfrequencies of the free oscillation modes $g^{+}$of the component stars (Cowling 1941). These proximities lead to resonances which enhance the tidal motions of the mass elements and can have significant consequences for the observational properties of the binary.

Zahn (1970) studied resonances of dynamic tides with low-frequency $g^{+}$-modes in non-rotating stars consisting of a convective core and a radiative envelope. For his purpose, he used an asymptotic representation for the

Send offprint requests to: B. Willems,

e-mail: b.willems@open.ac.uk eigenfunctions of the $g^{+}$-modes which he established neglecting the perturbation of the gravitational field due to the star's tidal distortion. Zahn's treatment was later generalised by Rocca (1987) to include the effects of the Coriolis force in slowly rotating binary components.

The luminosity variations associated with tidally excited oscillation modes in close binaries were investigated by Kumar et al. (1995). The authors proposed a new method for the determination of the orbital inclination based on fitting the shape of theoretically derived light curves to the shape of observationally determined light curves. They applied their results to various polytropic stellar models and to the binary pulsars PSR J0045-7319 and PSR B1259-63.

More recently, Smeyers et al. (1998) (hereafter referred to as Paper I) derived semi-analytical solutions for the components of a resonant dynamic tide by means of a two-time variable expansion procedure in which both the free and the forced oscillations of the star were treated as linear, isentropic oscillations of a spherically symmetric star. The authors concluded that, at the lowest-order of approximation, the oscillation mode involved in the resonance is excited with the forcing frequency of the resonant dynamic tide. Their investigation was subsequently 
extended by Willems et al. (2001) to take into account the effects of radiative damping in the nonadiabatic surface layers of a star.

The duration of a resonance is determined by the combined effect of stellar and orbital evolution. Witte \& Savonije $(1999,2001)$ have shown that when both effects are taken into account, a dynamic tide can easily become locked in a resonance for a prolonged period of time. Such a long-term resonance can have a significant influence on the secular evolution of the orbital elements and increases the probability of detecting the tidally induced oscillations in the star.

From an observational point of view, firm evidence of tidally excited oscillations is still scarce, possibly due to the absence of any systematic observational study on the nature of the pulsations discovered in close binaries. Studies of individual stars are reported in the literature, e.g. $\sigma$ Sco (Fitch 1967), 14 Aur A (Fitch \& Wisniewski 1979), Spica (Smith 1985a,b), 16 Lac (Chapellier et al. 1995), V 539 Arae (Clausen 1996), among others, but the resonant nature of the modes was hardly ever proven. An introduction to a systematic observational study in the case of early-type binaries is given by Aerts et al. (1998). Their initiative resulted from a more general longterm systematic study of line-profile variations in $\beta \mathrm{Cep}$ stars and in slowly pulsating B stars, which led to the assessment that many of these types of pulsators belong to a close binary. Further evidence for the presence of B-type $g$-mode pulsators in close binaries was found by De Cat et al. (2000). An additional systematic observational project in this respect is the SEarch for FOrced Nonradial Oscillations (SEFONO) by Harmanec et al. (1997).

In this investigation, our aim is to determine the radialvelocity variations associated with resonantly excited oscillation modes in binaries with short orbital periods. To this end, we use semi-analytical solutions for the components of a resonant dynamic tide derived by Smeyers et al. (1998). We conclude our investigation by applying our results to the slowly pulsating B star HD 177863.

The plan of the paper is as follows. In Sects. 2 and 3, we present the basic assumptions adopted in our investigation and we decompose the tide-generating potential in terms of spherical harmonics and in Fourier series in terms of the companion's mean motion. In Sects. 4 and 5, we derive an expression for the radial-velocity variations associated with a resonant dynamic tide. The contributions of non-resonant dynamic tides to the tidally induced radialvelocity variations are determined in Sect. 6. In Sect. 7, we determine the total variation of a star's radial velocity due to the various resonant and non-resonant dynamic tides and we illustrate the role of the orbital eccentricity and the orbital inclination. In Sect. 8, we apply our results to a binary consisting of a $5 M_{\odot}$ zero-age main sequence star and a $1.4 M_{\odot}$ compact companion. In Sect. 9, the possibility of a resonantly excited oscillation mode in the slowly pulsating B star HD 177863 is investigated. The final section is devoted to concluding remarks.

\section{Basic assumptions}

Consider a close binary system of stars that are orbiting around each other in an unvarying Keplerian orbit with semi-major axis $a$ and orbital eccentricity $e$. The first star, with mass $M_{1}$ and radius $R_{1}$, is rotating uniformly around an axis perpendicular to the orbital plane in the sense of the orbital motion. The angular velocity $\boldsymbol{\Omega}$ is assumed to be low so that the effects of the Coriolis force and the centrifugal force can be neglected. The second star, with mass $M_{2}$, is treated as a point mass.

We start from the right-handed orthogonal frame of reference $C_{1} x^{\prime 1} x^{\prime 2} x^{\prime 3}$ introduced in Paper I. The origin of the frame of reference coincides with the mass centre $C_{1}$ of the uniformly rotating star and the $x^{\prime 1} x^{\prime 2}$-plane corresponds to the orbital plane of the binary. The directions of the $x^{\prime 1}$ - and the $x^{\prime 3}$-axis coincide with the direction from the star's mass centre to the periastron in the companion's relative orbit and to the direction of the star's angular velocity $\boldsymbol{\Omega}$, respectively.

As in Paper I, we pass on to an orthogonal frame of reference whose origin and $x^{\prime \prime 3}$-axis correspond to the origin and the $x^{\prime 3}$-axis of the frame of reference $C_{1} x^{\prime 1} x^{\prime 2} x^{\prime 3}$, but whose $x^{\prime \prime 1}$-axis and $x^{\prime \prime 2}$-axis are corotating with the star. We use this frame of reference to describe the nonradial oscillations induced in the star by the orbiting companion. With respect to the corotating frame of reference, we introduce the spherical coordinates $\boldsymbol{r}=(r, \theta, \phi)$. The transformation formulae from the Cartesian coordinates $x^{\prime 1}$, $x^{\prime 2}, x^{\prime 3}$ to the spherical coordinates $r, \theta, \phi$ are given by

$$
\left.\begin{array}{l}
x^{\prime 1}=r \sin \theta \cos (\phi+\Omega t), \\
x^{\prime 2}=r \sin \theta \sin (\phi+\Omega t), \\
x^{\prime 3}=r \cos \theta .
\end{array}\right\}
$$

In order to study the radial-velocity variations due to the tidal motions of the mass elements located at the star's surface, it is convenient to pass on to a frame of reference whose origin coincides with the mass centre of the tidally distorted star and whose polar axis is directed from the mass centre to the observer. We perform this transformation in two steps.

First, we introduce an orthogonal frame of reference $C_{1} \alpha^{1} \alpha^{2} \alpha^{3}$ whose origin and $\alpha^{3}$-axis coincide with the origin and the $x^{\prime 3}$-axis of the frame of reference $C_{1} x^{\prime 1} x^{\prime 2} x^{\prime 3}$. The direction of the $\alpha^{1}$-axis corresponds to the direction from the star's mass centre to the ascending node in the relative orbit of the companion.

Next, we pass on to an orthogonal frame of reference $C_{1} \alpha^{1} \alpha^{\prime 2} \alpha^{\prime 3}$ with the same origin and the same $\alpha^{11}$-axis as the frame of reference $C_{1} \alpha^{1} \alpha^{2} \alpha^{3}$, but with its $\alpha^{\prime 3}$ axis directed from the star's mass centre to the observer. The Cartesian coordinates $\alpha^{1}, \alpha^{\prime 2}, \alpha^{\prime 3}$ are related to the 


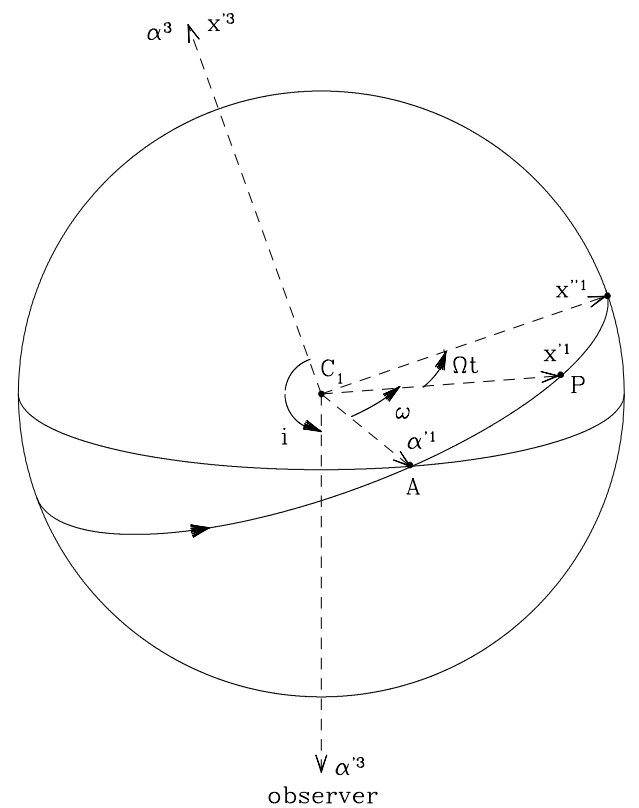

Fig. 1. The geometry involved in the introduction of the various frames of reference. The ascending node and the periastron of the relative orbit of the companion are denoted by the points labeled $\mathrm{A}$ and $\mathrm{P}$, respectively.

Cartesian coordinates $x^{\prime 1}, x^{\prime 2}, x^{\prime 3}$ by the transformation formulae

$$
\left(\begin{array}{c}
\alpha^{\prime 1} \\
\alpha^{\prime 2} \\
\alpha^{\prime 3}
\end{array}\right)=\left(\begin{array}{ccc}
\cos \omega & -\sin \omega & 0 \\
\sin \omega \cos i & \cos \omega \cos i & \sin i \\
-\sin \omega \sin i & -\cos \omega \sin i & \cos i
\end{array}\right)\left(\begin{array}{c}
x^{\prime 1} \\
x^{\prime 2} \\
x^{\prime 3}
\end{array}\right)
$$

Here $\omega$ is the longitude of the periastron in the relative orbit of the companion, and $i$ is the inclination of the orbital plane with respect to the plane perpendicular to the line of sight (see, e.g., Green 1985). With respect to the frame of reference $C_{1} \alpha^{\prime 1} \alpha^{\prime 2} \alpha^{\prime 3}$, we introduce the spherical coordinates $\boldsymbol{r}=\left(r^{\prime}, \theta^{\prime}, \phi^{\prime}\right)$.

The geometry involved in the introduction of the various frames of reference is illustrated in Fig. 1.

\section{The tide-generating potential}

The tidal force exerted by the companion is derived from the tide-generating potential $\varepsilon_{\mathrm{T}} W(\boldsymbol{r}, t)$, where $\varepsilon_{\mathrm{T}}$ is a small dimensionless parameter defined as

$\varepsilon_{\mathrm{T}}=\left(\frac{R_{1}}{a}\right)^{3} \frac{M_{2}}{M_{1}}$.

This parameter corresponds to the ratio of the tidal force to the gravity at the star's equator.

Following Polfliet \& Smeyers (1990), we expand the tide-generating potential in terms of unnormalised spherical harmonics $Y_{\ell}^{m}(\theta, \phi)$ and in Fourier series in terms of multiples of the companion's mean motion $n=2 \pi / T_{\text {orb }}$, where $T_{\text {orb }}$ is the orbital period. The expansion can be written as

$$
\begin{aligned}
\varepsilon_{\mathrm{T}} W(\boldsymbol{r}, t)= & \varepsilon_{\mathrm{T}} \sum_{\ell=2}^{4} \sum_{m=-\ell}^{\ell} \sum_{k=-\infty}^{\infty} W_{\ell, m, k}(\boldsymbol{r}) \\
& \times \exp \left[\mathrm{i}\left(\sigma_{\mathrm{T}} t-k n \tau\right)\right],
\end{aligned}
$$

where $\sigma_{\mathrm{T}}=k n+m \Omega$ is the forcing angular frequency with respect to the corotating frame of reference, $\tau$ is a time of periastron passage, and

$W_{\ell, m, k}(\boldsymbol{r})=-\frac{G M_{1}}{R_{1}} c_{\ell, m, k}\left(\frac{r}{R_{1}}\right)^{\ell} Y_{\ell}^{m}(\theta, \phi)$.

Here $G$ is the Newtonian constant of gravitation and $c_{\ell, m, k}$ a Fourier coefficient determined as

$$
\begin{aligned}
c_{\ell, m, k}= & \frac{(\ell-|m|) !}{(\ell+|m|) !} P_{\ell}^{|m|}(0)\left(\frac{R_{1}}{a}\right)^{\ell-2} \frac{1}{\left(1-e^{2}\right)^{\ell-1 / 2}} \\
& \times \frac{1}{\pi} \int_{0}^{\pi}(1+e \cos v)^{\ell-1} \cos (k M+m v) \mathrm{d} v .
\end{aligned}
$$

In the latter expression, $P_{\ell}^{m}(x)$ is an associated Legendre polynomial of the first kind, and $M$ and $v$ are respectively the mean and the true anomaly of the companion in its relative orbit.

The Fourier coefficients $c_{\ell, m, k}$ decrease with increasing values of the multiple $k$ of the companion's mean motion. The decrease is slower for higher orbital eccentricities, so that the number of terms that has to be taken into account in Eq. (4) of the tide-generating potential increases with increasing values of the orbital eccentricity. For $\ell=2$, the only non-zero coefficients $c_{2, m, k}$ are those associated with $m=-2,0,2$. A more elaborate discussion of these coefficients can be found in Willems (2000).

For realistic orbital periods, the forcing angular frequencies $\sigma_{\mathrm{T}}$ may be close to the eigenfrequencies of the star's free oscillation modes $g^{+}$. The tidal action exerted by the companion is then enhanced and the oscillation mode involved is resonantly excited with the forcing frequency of the dynamic tide (Paper I). The effects of resonant excitations of modes in close binaries are particularly important in the cases of resonances of dynamic tides with $g^{+}$-modes of a lower radial order.

\section{Tidal displacement due to resonant dynamic tides}

We consider a single partial dynamic tide generated by the term

$\varepsilon_{\mathrm{T}} W_{\ell, m, k}(\boldsymbol{r}) \exp \left[\mathrm{i}\left(\sigma_{\mathrm{T}} t-k n \tau\right)\right]$

in Eq. (4) of the tide-generating potential and assume the forcing angular frequency $\sigma_{\mathrm{T}}$ to be close to the eigenfrequency $\sigma_{\ell, N}$ of a free isentropic oscillation mode of radial order $N$ that is associated with the spherical harmonic $Y_{\ell}^{m}(\theta, \phi)$. For the sake of simplification, we introduce the abbreviation $S=(\ell, m, N)$ for the wave numbers $\ell, m$, and $N$ identifying the oscillation mode involved in the resonance. 
Furthermore, let the relative frequency difference

$\varepsilon=\frac{\sigma_{\ell, N}-\sigma_{\mathrm{T}}}{\sigma_{\ell, N}}$

be of the order of the ratio $\varepsilon_{\mathrm{T}}$ of the tidal force to the gravity at the star's equator. At the lowest-order of approximation, the components of the resonant dynamic tide with respect to the local coordinate basis $\partial / \partial r, \partial / \partial \theta, \partial / \partial \phi$ are then given by

$$
\left.\begin{array}{l}
(\delta r)_{\mathrm{T}}(\boldsymbol{r}, t)=\frac{1}{\varepsilon} \frac{\varepsilon_{\mathrm{T}}}{2} c_{\ell, m, k} Q_{\ell, N} \xi_{\ell, N}(r) \\
\times P_{\ell}^{|m|}(\cos \theta) \exp \left[\mathrm{i}\left(m \phi+\sigma_{\mathrm{T}} t-k n \tau\right)\right], \\
(\delta \theta)_{\mathrm{T}}(\boldsymbol{r}, t)=\frac{1}{\varepsilon} \frac{\varepsilon_{\mathrm{T}}}{2} c_{\ell, m, k} Q_{\ell, N} \frac{\eta_{\ell, N}(r)}{r^{2}} \\
\quad \times \frac{\partial P_{\ell}^{|m|}(\cos \theta)}{\partial \theta} \exp \left[\mathrm{i}\left(m \phi+\sigma_{\mathrm{T}} t-k n \tau\right)\right], \\
(\delta \phi)_{\mathrm{T}}(\boldsymbol{r}, t)=\frac{1}{\varepsilon} \frac{\varepsilon_{\mathrm{T}}}{2} c_{\ell, m, k} Q_{\ell, N} \frac{\eta_{\ell, N}(r)}{r^{2}} \\
\quad \times \frac{\mathrm{i} m}{\sin ^{2} \theta} P_{\ell}^{|m|}(\cos \theta) \exp \left[\mathrm{i}\left(m \phi+\sigma_{\mathrm{T}} t-k n \tau\right)\right]
\end{array}\right\}
$$

[Paper I, Eqs. (57)]. Here $\xi_{\ell, N}(r)$ and $\eta_{\ell, N}(r)$ are the radial parts of the radial and the transverse component of the Lagrangian displacement of the oscillation mode $S$ [Paper I, Eqs. (43)], and $Q_{\ell, N}$ is related to the work done by the tidal force through the oscillation mode $S$ [Paper I, Eq. (50)].

The components of the resonant dynamic tide with respect to the local coordinate basis $\partial / \partial r^{\prime}, \partial / \partial \theta^{\prime}, \partial / \partial \phi^{\prime}$ are obtained from Solutions (9) by application of the transformation formulae for contravariant vector components. The transformation formula takes the form

$$
\left(\begin{array}{c}
\left(\delta r^{\prime}\right)_{\mathrm{T}} \\
\left(\delta \theta^{\prime}\right)_{\mathrm{T}} \\
\left(\delta \phi^{\prime}\right)_{\mathrm{T}}
\end{array}\right)=\left(\begin{array}{lll}
\frac{\partial r^{\prime}}{\partial r} & \frac{\partial r^{\prime}}{\partial \theta} & \frac{\partial r^{\prime}}{\partial \phi} \\
\frac{\partial \theta^{\prime}}{\partial r} & \frac{\partial \theta^{\prime}}{\partial \theta} & \frac{\partial \theta^{\prime}}{\partial \phi} \\
\frac{\partial \phi^{\prime}}{\partial r} & \frac{\partial \phi^{\prime}}{\partial \theta} & \frac{\partial \phi^{\prime}}{\partial \phi}
\end{array}\right)\left(\begin{array}{c}
(\delta r)_{\mathrm{T}} \\
(\delta \theta)_{\mathrm{T}} \\
(\delta \phi)_{\mathrm{T}}
\end{array}\right)
$$

where

$$
\begin{aligned}
& \frac{\partial r^{\prime}}{\partial r}=1, \\
& \frac{\partial \theta^{\prime}}{\partial \theta}=\frac{\cos i-\sin i \sin \phi^{\prime} \cot \theta^{\prime}}{D\left(\theta^{\prime}, \phi^{\prime}\right)} \\
& \frac{\partial \theta^{\prime}}{\partial \phi}=\sin i \cos \phi^{\prime} \\
& \frac{\partial \phi^{\prime}}{\partial \theta}=-\frac{\sin i \cos \phi^{\prime}}{\sin ^{2} \theta^{\prime} D\left(\theta^{\prime}, \phi^{\prime}\right)} \\
& \frac{\partial \phi^{\prime}}{\partial \phi}=\cos i-\sin i \sin \phi^{\prime} \cot \theta^{\prime}
\end{aligned}
$$

and the other partial derivatives are equal to zero. The function $D\left(\theta^{\prime}, \phi^{\prime}\right)$ is given by

$D=\left[\cos ^{2} \phi^{\prime}+\left(\cos i \sin \phi^{\prime}-\sin i \cot \theta^{\prime}\right)^{2}\right]^{1 / 2}$.
In addition, the spherical harmonics $Y_{\ell}^{m}(\theta, \phi)$ are transformed according to

$$
\begin{aligned}
Y_{\ell}^{m}(\theta, \phi)= & \exp [-\mathrm{i} m(\omega+\Omega t+\pi / 2)] \\
& \times \sum_{k^{\prime}=-\ell}^{\ell} a_{\ell, m, k^{\prime}}(i) Y_{\ell}^{k^{\prime}}\left(\theta^{\prime}, \phi^{\prime}\right),
\end{aligned}
$$

where the coefficients $a_{\ell, m, k^{\prime}}(i)$ are functions of the orbital inclination defined as

$$
\begin{aligned}
& a_{\ell, m, k^{\prime}}(i)=\mu_{m}^{-1} \mu_{k^{\prime}}(\ell+m) !\left(\ell-k^{\prime}\right) ! \exp \left(\mathrm{i} k^{\prime} \pi / 2\right) \\
& \quad \times \sum_{j} \frac{(-1)^{\ell-m-j}\left(\cos \frac{i}{2}\right)^{2 j+m+k^{\prime}}\left(\sin \frac{i}{2}\right)^{2 \ell-2 j-m-k^{\prime}}}{j !(\ell-m-j) !\left(\ell-k^{\prime}-j\right) !\left(m+k^{\prime}+j\right) !}
\end{aligned}
$$

(Jeffreys 1965). In this definition, the summation is performed over all values of $j$ satisfying the relations

$j \geq 0, j \geq-m-k^{\prime}, j \leq \ell-m, j \leq \ell-k^{\prime}$,

and the factors $\mu_{k^{\prime}}$ are given by

$$
\left.\begin{array}{ll}
\mu_{k^{\prime}}=1 & \text { for } k^{\prime} \geq 0, \\
\mu_{k^{\prime}}=(-1)^{k^{\prime}} \frac{\left(\ell+k^{\prime}\right) !}{\left(\ell-k^{\prime}\right) !} & \text { for } k^{\prime}<0 .
\end{array}\right\}
$$

At the lowest order of approximation, the components of the resonant dynamic tide with respect to the local coordinate basis $\partial / \partial r^{\prime}, \partial / \partial \theta^{\prime}, \partial / \partial \phi^{\prime}$ then take the form

$$
\begin{aligned}
& \left(\delta r^{\prime}\right)_{\mathrm{T}}\left(\boldsymbol{r}^{\prime}, t\right)=\frac{1}{\varepsilon} \frac{\varepsilon_{\mathrm{T}}}{2} c_{\ell, m, k} Q_{\ell, N} \xi_{\ell, N}\left(r^{\prime}\right) \\
& \times \sum_{k^{\prime}=-\ell}^{\ell} a_{\ell, m, k^{\prime}}(i) P_{\ell}^{\left|k^{\prime}\right|}\left(\cos \theta^{\prime}\right) \\
& \times \exp \left\{\mathrm{i}\left[k^{\prime} \phi^{\prime}+k M-m\left(\omega+\frac{\pi}{2}\right)\right]\right\}, \\
& \left(\delta \theta^{\prime}\right)_{\mathrm{T}}\left(\boldsymbol{r}^{\prime}, t\right)=\frac{1}{\varepsilon} \frac{\varepsilon_{\mathrm{T}}}{2} c_{\ell, m, k} Q_{\ell, N} \frac{\eta_{\ell, N}\left(r^{\prime}\right)}{r^{\prime 2}} \\
& \times \sum_{k^{\prime}=-\ell}^{\ell} a_{\ell, m, k^{\prime}}(i) \frac{\partial P_{\ell}^{\left|k^{\prime}\right|}\left(\cos \theta^{\prime}\right)}{\partial \theta^{\prime}} \\
& \times \exp \left\{\mathrm{i}\left[k^{\prime} \phi^{\prime}+k M-m\left(\omega+\frac{\pi}{2}\right)\right]\right\}, \\
& \left(\delta \phi^{\prime}\right)_{\mathrm{T}}\left(\boldsymbol{r}^{\prime}, t\right)=\frac{1}{\varepsilon} \frac{\varepsilon_{\mathrm{T}}}{2} c_{\ell, m, k} Q_{\ell, N} \frac{\eta_{\ell, N}\left(r^{\prime}\right)}{r^{\prime 2} \sin ^{2} \theta^{\prime}} \\
& \quad \times \sum_{k^{\prime}=-\ell}^{\ell} a_{\ell, m, k^{\prime}}(i) \mathrm{i} k^{\prime} P_{\ell}^{\left|k^{\prime}\right|}\left(\cos \theta^{\prime}\right) \\
& \quad \times \exp \left\{\mathrm{i}\left[k^{\prime} \phi^{\prime}+k M-m\left(\omega+\frac{\pi}{2}\right)\right]\right\} .
\end{aligned}
$$

\section{Radial-velocity variations associated with resonant dynamic tides}

The tidal motions of the mass elements located at the star's surface contribute to the radial-velocity variations seen by the observer. Since the mass elements of the undistorted equilibrium star are assumed to be at rest with 
respect to the corotating frame of reference, the radial component of the velocity field associated with the tidal motions is given by

$v_{\mathrm{T}}=\frac{\partial(\delta \boldsymbol{r})_{\mathrm{T}}}{\partial t} \cdot\left(-\boldsymbol{e}_{\alpha^{\prime 3}}\right)=\mathrm{i} \sigma_{\mathrm{T}}(\delta \boldsymbol{r})_{\mathrm{T}} \cdot\left(-\boldsymbol{e}_{\alpha^{\prime 3}}\right)$,

where

$\boldsymbol{e}_{\alpha^{\prime 3}}=\cos \theta^{\prime} \frac{\partial}{\partial r^{\prime}}-\frac{\sin \theta^{\prime}}{r^{\prime}} \frac{\partial}{\partial \theta^{\prime}}$

is a unit vector in the direction of the $\alpha^{\prime 3}$-axis.

In the case of a resonance between a dynamic tide and a free oscillation mode, the tidally induced radial-velocity variations due to the mass elements located at the star's surface take the form

$$
\begin{aligned}
& v_{\mathrm{T} ; \text { res }}\left(R_{1}, \theta^{\prime}, \phi^{\prime} ; t\right)=-\frac{1}{\varepsilon} \frac{\varepsilon_{\mathrm{T}}}{2} c_{\ell, m, k} Q_{\ell, N} \mathrm{i} \sigma_{\mathrm{T}} \\
& \quad \times \sum_{k^{\prime}=-\ell}^{\ell} a_{\ell, m, k^{\prime}}(i)\left[\cos \theta^{\prime} \xi_{\ell, N}\left(R_{1}\right) P_{\ell}^{\left|k^{\prime}\right|}\left(\cos \theta^{\prime}\right)\right. \\
& \left.\quad-\sin \theta^{\prime} \frac{\eta_{\ell, N}\left(R_{1}\right)}{R_{1}} \frac{\partial P_{\ell}^{\left|k^{\prime}\right|}\left(\cos \theta^{\prime}\right)}{\partial \theta^{\prime}}\right] \\
& \quad \times \exp \left\{\mathrm{i}\left[k^{\prime} \phi^{\prime}+k M-m\left(\omega+\frac{\pi}{2}\right)\right]\right\} .
\end{aligned}
$$

The velocity variations seen by the observer are obtained by taking the average of Eq. (19) over the visible hemisphere of the star. When one neglects the distortion of the stellar surface and the perturbation of the specific intensity due to the tidal motions of the mass elements, the average is determined as

$$
\begin{aligned}
& V_{\text {T; res }}(t)= \\
& \frac{\int_{0}^{2 \pi} \int_{0}^{\pi / 2} h\left(\theta^{\prime}\right) v_{\text {T;res }}\left(R_{1}, \theta^{\prime}, \phi^{\prime} ; t\right) \sin \theta^{\prime} \cos \theta^{\prime} \mathrm{d} \theta^{\prime} \mathrm{d} \phi^{\prime}}{\int_{0}^{2 \pi} \int_{0}^{\pi / 2} h\left(\theta^{\prime}\right) \sin \theta^{\prime} \cos \theta^{\prime} \mathrm{d} \theta^{\prime} \mathrm{d} \phi^{\prime}}
\end{aligned}
$$

where $h\left(\theta^{\prime}\right)$ is a limb-darkening law of the form

$h\left(\theta^{\prime}\right)=1-u+u \cos \theta^{\prime}, \quad u \in[0,1]$.

After performing the integration over the azimuthal angle $\phi^{\prime}$, one obtains

$$
\begin{aligned}
V_{\mathrm{T} ; \mathrm{res}}(t)= & -\frac{1}{\varepsilon} \frac{\varepsilon_{\mathrm{T}}}{2} c_{\ell, m, k} \mathrm{i} \sigma_{\mathrm{T}} a_{\ell, m, 0}(i) \\
& \times\left[f_{\xi, \ell}(u) \xi_{\ell, N}\left(R_{1}\right)-f_{\eta, \ell}(u) \frac{\eta_{\ell, N}\left(R_{1}\right)}{R_{1}}\right] Q_{\ell, N} \\
& \times \exp \left\{\mathrm{i}\left[k M-m\left(\omega+\frac{\pi}{2}\right)\right]\right\} .
\end{aligned}
$$

Here $f_{\xi, \ell}(u)$ and $f_{\eta, \ell}(u)$ are functions of the limbdarkening coefficient $u$ defined as

$$
\begin{aligned}
& f_{\xi, \ell}(u)=\frac{6}{3-u} \int_{0}^{\pi / 2} h\left(\theta^{\prime}\right) P_{\ell}\left(\cos \theta^{\prime}\right) \sin \theta^{\prime} \cos ^{2} \theta^{\prime} \mathrm{d} \theta^{\prime}, \\
& f_{\eta, \ell}(u)=\frac{6}{3-u} \int_{0}^{\pi / 2} h\left(\theta^{\prime}\right) \frac{\partial P_{\ell}\left(\cos \theta^{\prime}\right)}{\partial \theta^{\prime}} \sin ^{2} \theta^{\prime} \cos \theta^{\prime} \mathrm{d} \theta^{\prime} .
\end{aligned}
$$

The functions are described in more detail in Appendix A.
In addition to the partial dynamic tide with forcing angular frequency $\sigma_{\mathrm{T}}$, we also take into account the partial dynamic tide with forcing angular frequency $-\sigma_{\mathrm{T}}$, which is also resonant. By the use of the symmetry properties $c_{\ell,-m,-k}=c_{\ell, m, k}$ and $a_{\ell,-m, 0}=a_{\ell, m, 0}$, the global solution for the variation of the star's radial velocity due to a resonance of a dynamic tide with a free oscillation mode takes the form

$V_{\mathrm{T} ; \mathrm{res}}(t)=\varepsilon_{\mathrm{T}} a_{\ell, m, 0}(i) c_{\ell, m, k}(e) V_{\mathrm{T} ; \mathrm{res}}^{*}(t)$,

where

$$
\begin{aligned}
V_{\mathrm{T} ; \mathrm{res}}^{*}(t)= & \frac{1}{\varepsilon} \sigma_{\mathrm{T}}\left[f_{\xi, \ell}(u) \xi_{\ell, N}\left(R_{1}\right)-f_{\eta, \ell}(u) \frac{\eta_{\ell, N}\left(R_{1}\right)}{R_{1}}\right] \\
& \times Q_{\ell, N} \sin \left[k n(t-\tau)-m\left(\omega+\frac{\pi}{2}\right)\right] .
\end{aligned}
$$

In this expression, the products $Q_{\ell, N} \xi_{\ell, N}\left(R_{1}\right)$ and $Q_{\ell, N} \eta_{\ell, N}\left(R_{1}\right)$ are independent of the normalisation adopted for the determination of the free oscillation mode of degree $\ell$ and radial order $N$ that is involved in the resonance. They also decrease rapidly with increasing values of $N$ so that the influence of higher-order modes on the tidally induced radial-velocity variations is substantially smaller than that of lower-order modes (Willems et al. 1997; Willems 2000).

\section{Radial-velocity variations associated with non-resonant dynamic tides}

We again consider a single partial dynamic tide generated by a term of the form

$\varepsilon_{\mathrm{T}} W_{\ell, m, k}(\boldsymbol{r}) \exp \left[\mathrm{i}\left(\sigma_{\mathrm{T}} t-k n \tau\right)\right]$

in Eq. (4) of the tide-generating potential, but now assume the forcing angular frequency $\sigma_{\mathrm{T}}$ to be far from any of the eigenfrequencies associated with the star's free isentropic oscillation modes. The components of the tidal displacement field generated by this term can be expressed as

$$
\begin{aligned}
& (\delta r)_{\mathrm{T}}(\boldsymbol{r}, t)=\varepsilon_{\mathrm{T}} c_{\ell, m, k} \xi_{\mathrm{T}}(r) \\
& \quad \times P_{\ell}^{|m|}(\cos \theta) \exp \left[\mathrm{i}\left(m \phi+\sigma_{\mathrm{T}} t-k n \tau\right)\right], \\
& (\delta \theta)_{\mathrm{T}}(\boldsymbol{r}, t)=\varepsilon_{\mathrm{T}} c_{\ell, m, k} \frac{\eta_{\mathrm{T}}(r)}{r^{2}} \\
& \quad \times \frac{\partial P_{\ell}^{|m|}(\cos \theta)}{\partial \theta} \exp \left[\mathrm{i}\left(m \phi+\sigma_{\mathrm{T}} t-k n \tau\right)\right], \\
& (\delta \phi)_{\mathrm{T}}(\boldsymbol{r}, t)=\varepsilon_{\mathrm{T}} c_{\ell, m, k} \frac{\eta_{\mathrm{T}}(r)}{r^{2}} \frac{\mathrm{i} m}{\sin ^{2} \theta} \\
& \quad \times P_{\ell}^{|m|}(\cos \theta) \exp \left[\mathrm{i}\left(m \phi+\sigma_{\mathrm{T}} t-k n \tau\right)\right],
\end{aligned}
$$

where $\xi_{\mathrm{T}}(r)$ and $\eta_{\mathrm{T}}(r)$ are solutions of the homogeneous fourth-order system of linear differential equations

$$
\frac{\mathrm{d}\left(r^{2} \xi_{\mathrm{T}}\right)}{\mathrm{d} r}=\frac{g}{c^{2}} r^{2} \xi_{\mathrm{T}}+\left[\ell(\ell+1)-\sigma_{\mathrm{T}}^{2} \frac{r^{2}}{c^{2}}\right] \eta_{\mathrm{T}}+\frac{r^{2}}{c^{2}} \Psi_{\mathrm{T}},
$$




$$
\begin{aligned}
& \frac{\mathrm{d} \eta_{\mathrm{T}}}{\mathrm{d} r}=\left(1-\frac{N_{\mathrm{b}}^{2}}{\sigma_{\mathrm{T}}^{2}}\right) \xi_{\mathrm{T}}+\frac{N_{\mathrm{b}}^{2}}{g} \eta_{\mathrm{T}}-\frac{1}{\sigma_{\mathrm{T}}^{2}} \frac{N_{\mathrm{b}}^{2}}{g} \Psi_{\mathrm{T}}, \\
& \frac{1}{r^{2}} \frac{\mathrm{d}}{\mathrm{d} r}\left(r^{2} \frac{\mathrm{d} \Psi_{\mathrm{T}}}{\mathrm{d} r}\right)-\frac{\ell(\ell+1)}{r^{2}} \Psi_{\mathrm{T}}=4 \pi G \rho \\
& \times\left[\frac{N_{\mathrm{b}}^{2}}{g} \xi_{\mathrm{T}}+\frac{1}{c^{2}}\left(\sigma_{\mathrm{T}}^{2} \eta_{\mathrm{T}}-\Psi_{\mathrm{T}}\right)\right] .
\end{aligned}
$$

$$
\left.\begin{array}{l}
a_{2,0,0}(i)=\frac{1}{2}\left(3 \cos ^{2} i-1\right), \\
a_{2,2,0}(i)=a_{2,-2,0}(i)=3 \sin ^{2} i .
\end{array}\right\}
$$

Here $\Psi_{\mathrm{T}}(r)$ is the radial part of the total perturbation of the gravitational potential (for a definition see, e.g., Polfliet \& Smeyers 1990), $g$ the local gravity, $c^{2}$ the square of the isentropic sound velocity, and $N_{\mathrm{b}}^{2}$ the square of the Brunt-Väisälä frequency. The functions $\xi_{\mathrm{T}}(r), \eta_{\mathrm{T}}(r)$, and $\Psi_{\mathrm{T}}(r)$ depend on the values of the azimuthal number $m$ and on the Fourier index $k$ through the forcing angular frequency $\sigma_{\mathrm{T}}$.

The solutions of Eqs. (29)-(31) must satisfy boundary conditions at the star's centre and at the star's surface. At $r=0$, the radial component of the tidal displacement must remain finite. At $r=R_{1}$, the Lagrangian perturbation of the pressure must vanish, and the continuity of the gravitational potential and its gradient requires that

$$
\begin{aligned}
\left(\frac{\mathrm{d} \Psi_{\mathrm{T}}}{\mathrm{d} r}\right)_{R_{1}} & +\frac{\ell+1}{R_{1}}\left(\Psi_{\mathrm{T}}\right)_{R_{1}}+\left(4 \pi G \rho \xi_{\mathrm{T}}\right)_{R_{1}} \\
& =-(2 \ell+1) \times \frac{G M_{1}}{R_{1}^{2}} .
\end{aligned}
$$

By comparison of Eqs. (28) with Eqs. (9), it follows that the contribution of a non-resonant dynamic tide to the tidally induced radial-velocity variations takes the form

$$
\begin{aligned}
& V_{\mathrm{T} ; \ell, m, k}(t)=\varepsilon_{\mathrm{T}} a_{\ell, m, 0}(i) c_{\ell, m, k}(e) V_{\mathrm{T} ; \ell, m, k}^{*}(t) \\
& \text { with } \\
& V_{\mathrm{T} ; \ell, m, k}^{*}(t)= \\
& \qquad 2 \sigma_{\mathrm{T}}\left[f_{\xi, \ell}(u) \xi_{\mathrm{T} ; \ell, m, k}\left(R_{1}\right)-f_{\eta, \ell}(u) \frac{\eta_{\mathrm{T} ; \ell, m, k}\left(R_{1}\right)}{R_{1}}\right] \\
& \quad \times \sin \left[k n(t-\tau)-m\left(\omega+\frac{\pi}{2}\right)\right] .
\end{aligned}
$$

\section{Total variation of a star's radial velocity due to its tidal response}

In accordance with Eq. (4) of the tide-generating potential, the total variation of the star's radial velocity due to the tidal motions of the mass elements located at its surface, is obtained by adding the contributions stemming from the various resonant and non-resonant partial dynamic tides.

When one restricts the contributions to those resulting from the tides associated with the second-degree spherical harmonics, the total variation of the star's radial velocity due to its tidal response can be written as

$$
\begin{aligned}
& V_{\mathrm{T} ; \operatorname{rad}}(t)=\varepsilon_{\mathrm{T}} \sum_{k=1}^{\infty}\left\{a_{2,0,0}(i) c_{2,0, k}(e) V_{\mathrm{T} ; 2,0, k}^{*}(t)\right. \\
& \left.\quad+a_{2,2,0}(i)\left[c_{2,-2, k}(e) V_{\mathrm{T} ; 2,-2, k}^{*}(t)+c_{2,2, k}(e) V_{\mathrm{T} ; 2,2, k}^{*}(t)\right]\right\},
\end{aligned}
$$

where

In the case of a resonance of a dynamic tide characterised by the azimuthal number $m$ and the Fourier index $k$ with a free oscillation mode of radial order $N$, the factor $V_{\mathrm{T} ; 2, m, k}^{*}(t)$ must be replaced by the factor $V_{\mathrm{T} ; \mathrm{res}}^{*}(t)$.

The contributions of the various partial dynamic tides to the observed tidally induced radial-velocity variations depend on the orbital inclination and on the orbital eccentricity through the coefficients $a_{2, m, 0}(i)$ and $c_{2, m, k}(e)$, respectively. The variation of the product of the coefficients $a_{2, m, 0}(i)$ and $c_{2, m, k}(e)$ is displayed in Fig. 2 as a function of $k$, for the orbital inclinations $i=5^{\circ}$ and $i=85^{\circ}$, and the orbital eccentricities $e=0.3$ and $e=0.5$. The product decreases with increasing values of $k$ due to the rapid decrease of the Fourier coefficients $c_{2, m, k}(e)$ for higher-order harmonics in Eq. (4) of the tide-generating potential.

In the case of the lower orbital inclination $i=5^{\circ}$, the products of the coefficients $a_{2, m, 0}(i)$ and $c_{2, m, k}(e)$ are largest in absolute value for the coefficients associated with the azimuthal number $m=0$. The prevalence is more pronounced for the eccentricity $e=0.5$ than for the eccentricity $e=0.3$ due to the increase of the coefficients $c_{2,0, k}(e)$ with increasing values of the orbital eccentricity. For larger values of the orbital inclination, the products of the coefficients $a_{2, m, 0}(i)$ and $c_{2, m, k}(i)$ associated with $m=-2$ rapidly become the dominant products. In the case of the orbital inclination $i=85^{\circ}$, they are approximately two orders of magnitude larger in absolute value than the products associated with the azimuthal number $m=0$. The products of the coefficients associated with $m=2$ are generally two or more orders of magnitude smaller than the products associated with the azimuthal numbers $m=-2$ and $m=0$.

\section{Tidally induced radial-velocity variations in a $5 M_{\odot}$ zero-age main sequence star}

We have applied the expressions derived in the previous sections to a binary consisting of a $5 M_{\odot}$ zero-age main sequence star and a $1.4 M_{\odot}$ companion which we approximate by a point mass. We considered orbital periods ranging from 2 to 6 days and the orbital eccentricities $e=0.3$ and $e=0.5$. The rotation of the star is assumed to be synchronised with the orbital motion of the companion in the periastron of its relative orbit. In addition, we have set both the longitude of the periastron $\omega$ and the time of periastron passage $\tau$ equal to zero. For the limb-darkening coefficient we adopted the value $u=0.36$, which is appropriate for main-sequence B-type stars.

The observed amplitude of the tidally induced radialvelocity variations is displayed in Figs. 3 and 4 as a function of the orbital period. The orbital inclination varies from $i=0^{\circ}$ in the upper panels of the figures to $i=90^{\circ}$ 

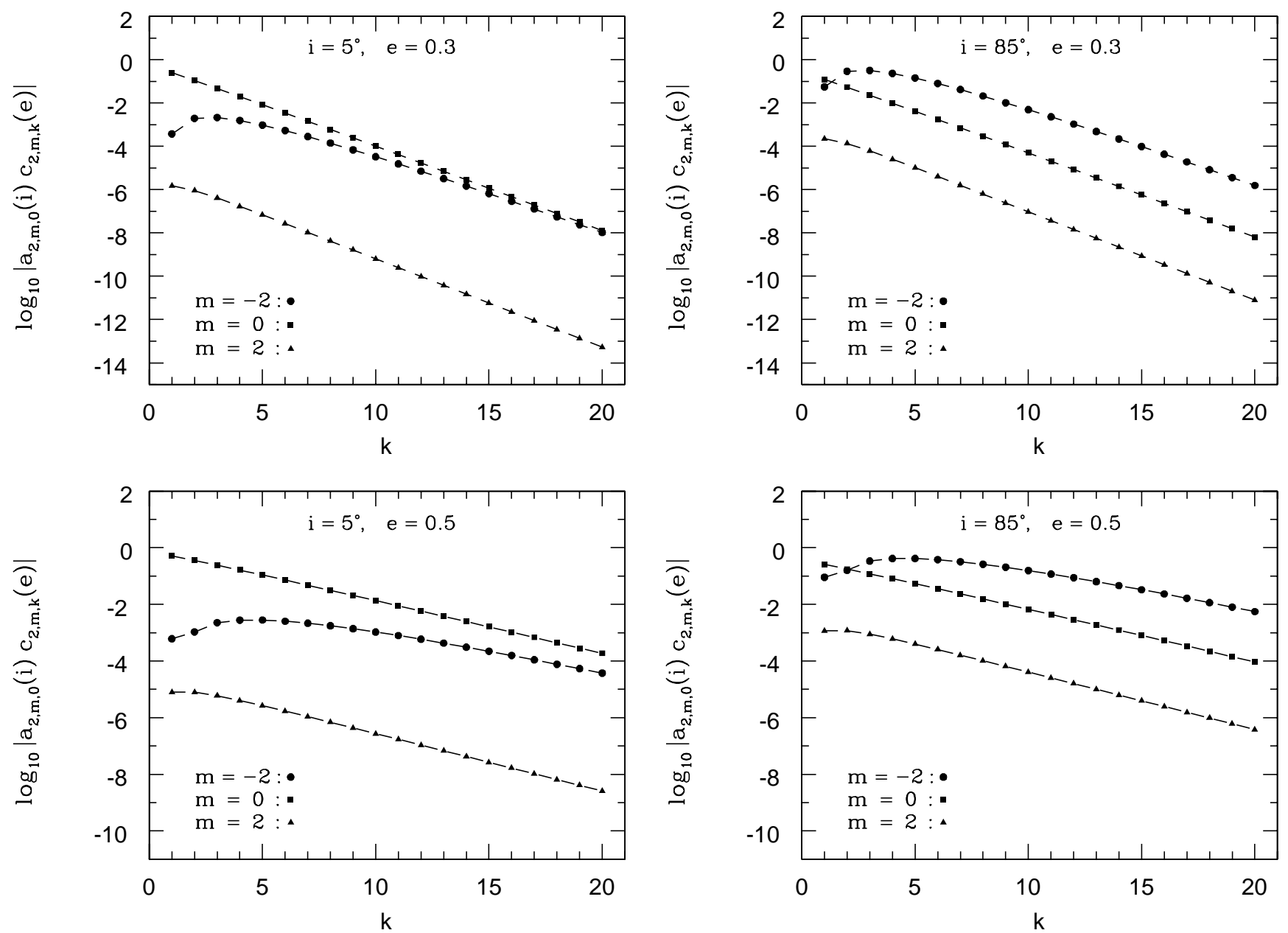

Fig. 2. Logarithmic representation of the absolute value of the products $a_{2, m, 0}(i) c_{2, m, k}(e)$ for the orbital inclinations $i=5^{\circ}$ (left-hand panels) and $i=85^{\circ}$ (right-hand panels), and the orbital eccentricities $e=0.3$ (top panels) and $e=0.5$ (bottom panels).

in the lower panels. The numerous peaks appearing at shorter orbital periods correspond to resonances of dynamic tides with free oscillation modes. Bearing in mind that, in the perturbation theory used in Paper I, the relative frequency difference $\varepsilon$ is assumed to be of the order of $\varepsilon_{\mathrm{T}}$, the calculations near the resonances are restricted to values of $\varepsilon$ larger than or equal to $0.1 \varepsilon_{\mathrm{T}}$ in absolute value.

When the orbital plane is observed face-on, i.e. when $i=0^{\circ}$, the tidally induced radial-velocity variations are determined solely by the dynamic tides associated with the azimuthal number $m=0$. For higher values of the orbital inclination, additional contributions arise from the dynamic tides associated with the azimuthal numbers $m= \pm 2$, although the contributions associated with $m=2$ are usually negligible. The amplitude of the tidally induced radial-velocity variations is largest when the orbital plane is seen edge-on, i.e. when $i=90^{\circ}$. The resonances are then almost exclusively due to the dynamic tides associated with the azimuthal number $m=-2$.

For close resonances, the amplitudes of the tidally induced radial-velocity variations can be quite large so that they are certainly detectable in observations. They increase with increasing values of the orbital eccentricity due to the smaller periastron distances associated with higher orbital eccentricities. The number of resonances is also larger for the orbital eccentricity $e=0.5$ than for the orbital eccentricity $e=0.3$ due to the larger number of terms that has to be taken into account in Eq. (4) of the tide-generating potential. For some cases our results point towards very high amplitudes which are above the typical sound speed in the atmosphere of the star. This would imply shock waves to occur, but our linear theory breaks down at these high velocities. The study of the nonlinear effects is currently beyond the scope of our investigation and the applications are limited to radial-velocity variations that can be well described by the linear theory.

A detailed representation of the effects of resonances in the range of orbital periods from 3.3 to 3.7 days is given in Fig. 5 for the orbital eccentricity $e=0.5$ and the orbital inclinations $i=20^{\circ}$ and $i=70^{\circ}$. The strongest resonances observed give rise to radial-velocity variations with amplitudes well above $5 \mathrm{~km} \mathrm{~s}^{-1}$. Several orbital periods lead to resonances with two oscillation modes simultaneously. 

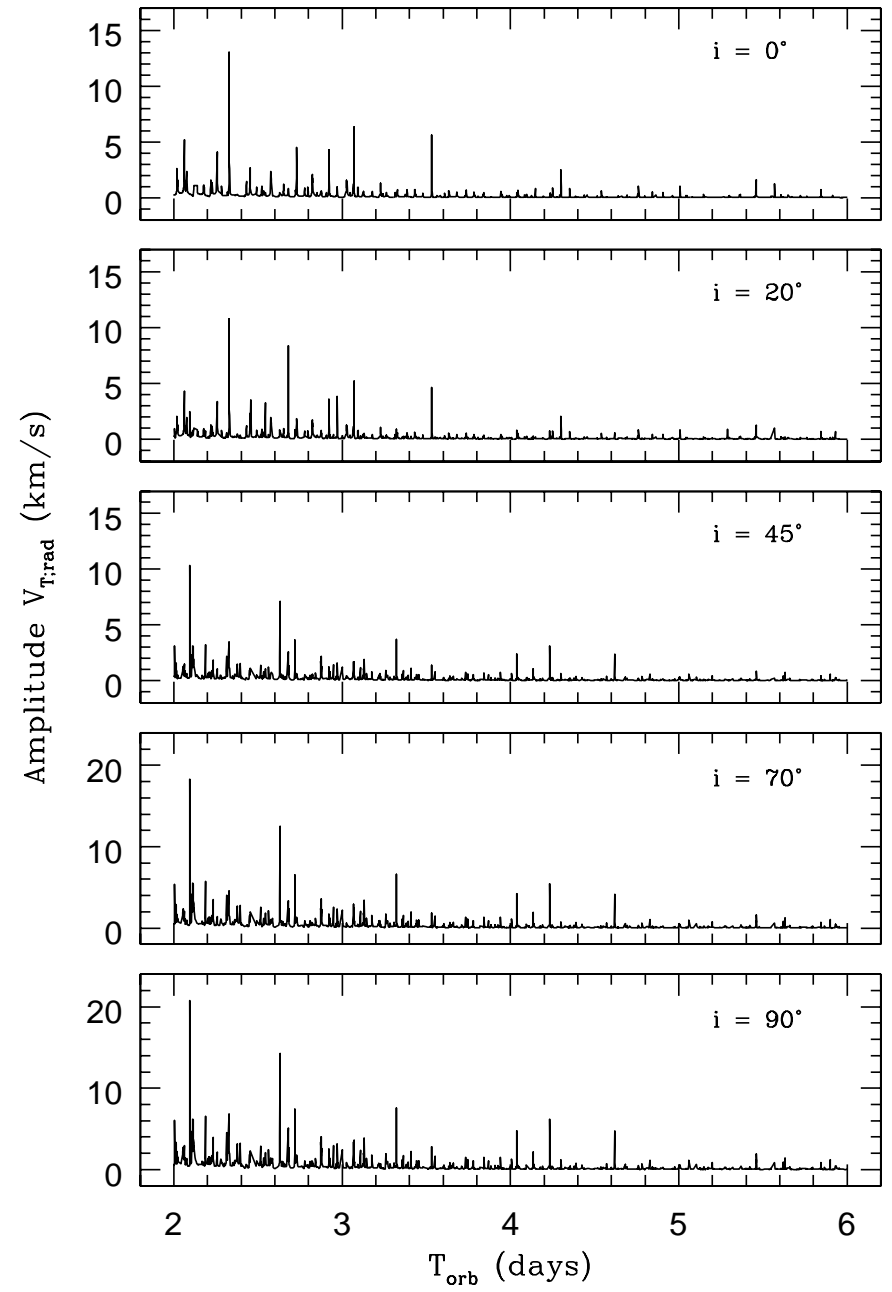

Fig. 3. The observed amplitude of the tidally induced radialvelocity variations as a function of the orbital period for the $5 M_{\odot}$ ZAMS stellar model and the orbital eccentricity $e=0.3$.

The peak in the amplitude near $T_{\text {orb }}=3.37$ days, for instance, is caused by simultaneous resonances with the oscillation modes $g_{12}^{+}$and $g_{16}^{+}$.

In Fig. 6, the tidally induced radial-velocity variations corresponding to the points labeled (a) - (e) are shown as a function of the orbital phase. The central panel shows that for an exact resonance - point (c), the radial-velocity variations are independent of the orbital phase. The reason is that the tidally induced radial-velocity variations given by Eq. (35) are dominated by the term corresponding to the resonant dynamic tide. Outside the resonance - cases (a) and (e), the radial-velocity variations are markedly less regular functions of the orbital phase since they are now the result of a superposition of purely non-resonant dynamic tides.

\section{Comparison with observations: HD 177863}

The bright slowly pulsating B star (SPB) HD 177863 was discovered to be a radial-velocity variable by Eggen (1977), while Waelkens \& Rufener (1985) detected intrinsic photometric variability in this object. Later on,
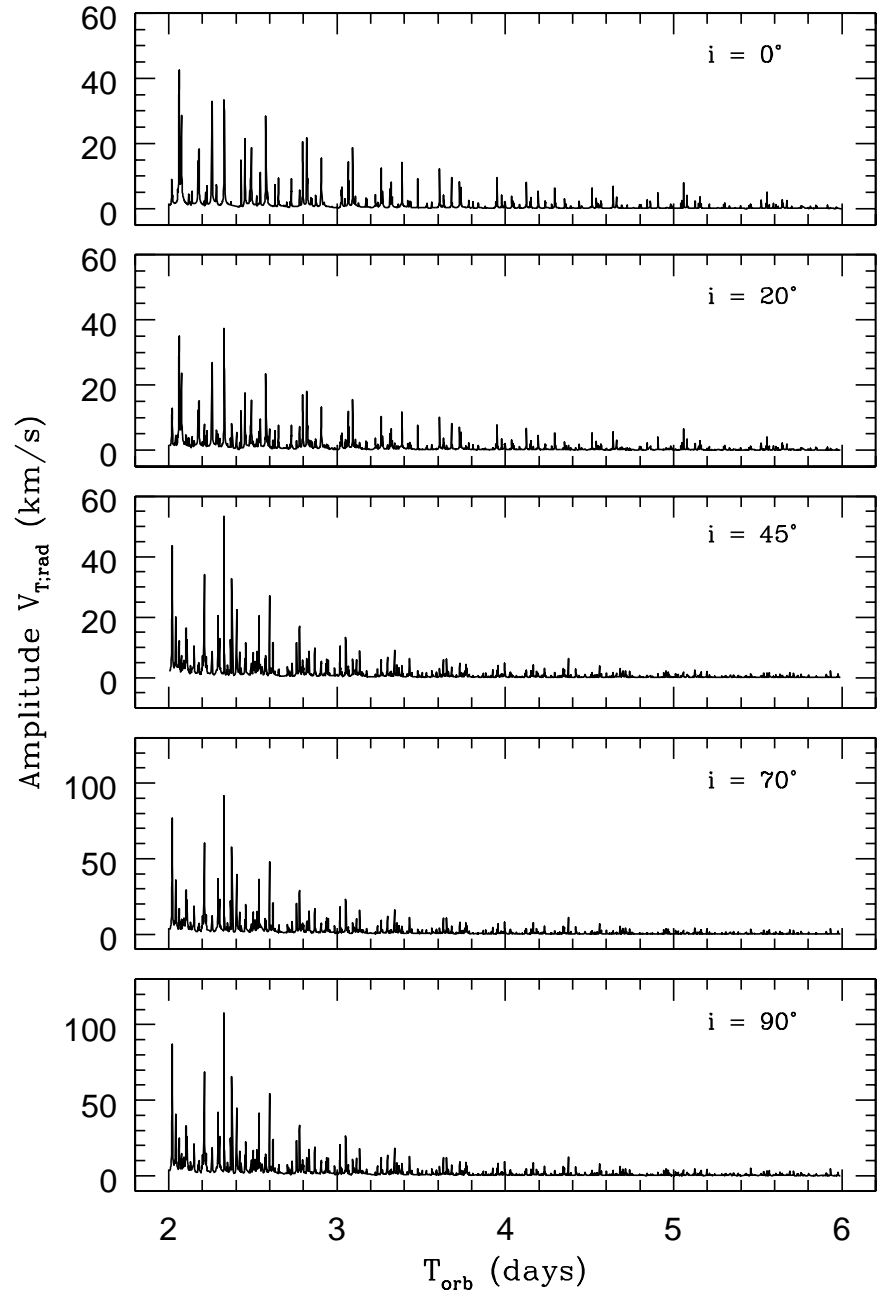

Fig. 4. The observed amplitude of the tidally induced radialvelocity variations as a function of the orbital period for the $5 M_{\odot}$ ZAMS stellar model and the orbital eccentricity $e=0.5$.

Waelkens (1991) classified the star as an SPB. De Cat et al. (2000) showed this star to be a single-lined spectroscopic binary and determined the orbital parameters of the system. They found an orbital period $T_{\text {orb }}=$ $11.9154 \pm 0.0009$ days, an eccentricity $e=0.60$, a longitude of the periastron $\varpi=182^{\circ}$, and a time of periastron passage $\tau=2450155.78 \mathrm{JD}$. The large eccentricity implies that the system is either still very young or that it has undergone some episodes of mass transfer. We find no indication of the occurrence of such episodes in the literature, while the relatively young age is confirmed by the study of Westin (1985), who finds an age of 64 million years. In what follows, we therefore assume that the system consists of two main-sequence stars. From the spectroscopic analysis and the mass function, one can furthermore derive that the orbital inclination is between 35 and 90 degrees and that the companion has a mass smaller than $2 M_{\odot}$. We also note that De Cat \& Aerts (in preparation) have found the primary to rotate supersynchronously with a projected equatorial rotation velocity between 45 and $60 \mathrm{~km} \mathrm{~s}^{-1}$.

De Cat (2001) also performed a detailed frequency study on both extensive spectroscopic and photometric 


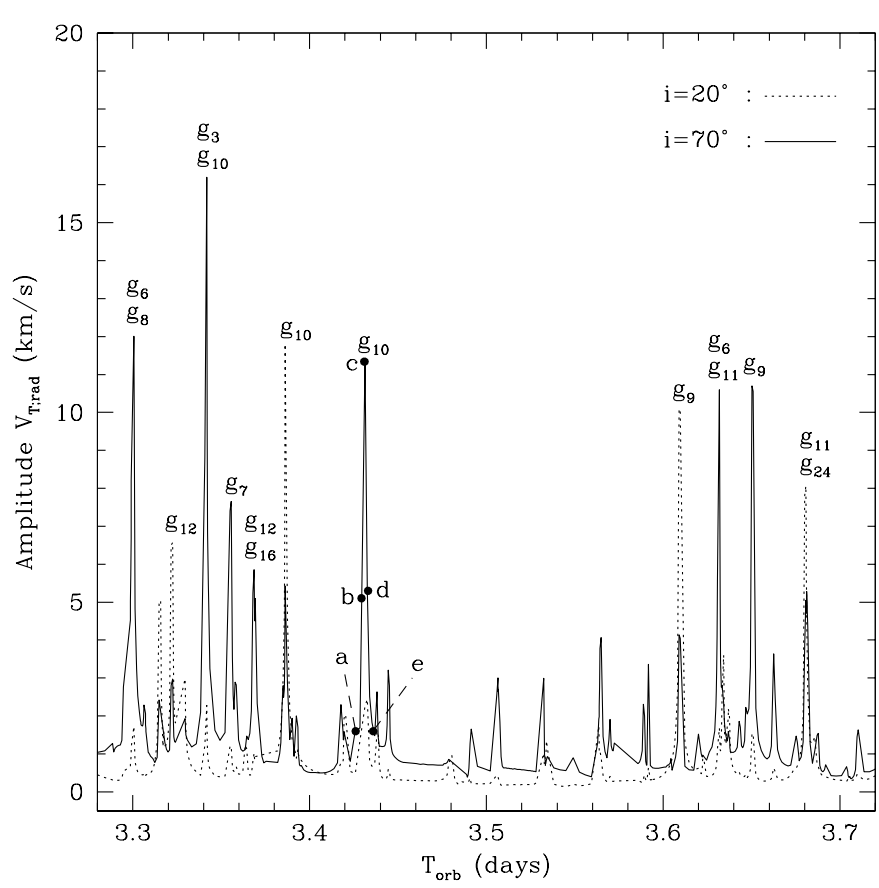

Fig. 5. Detailed representation of the observed amplitude of the tidally induced radial-velocity variations of the $5 M_{\odot}$ ZAMS stellar model for the orbital eccentricity $e=0.5$, and the orbital inclinations $i=20^{\circ}$ (dotted line) and $i=70^{\circ}$ (solid line).
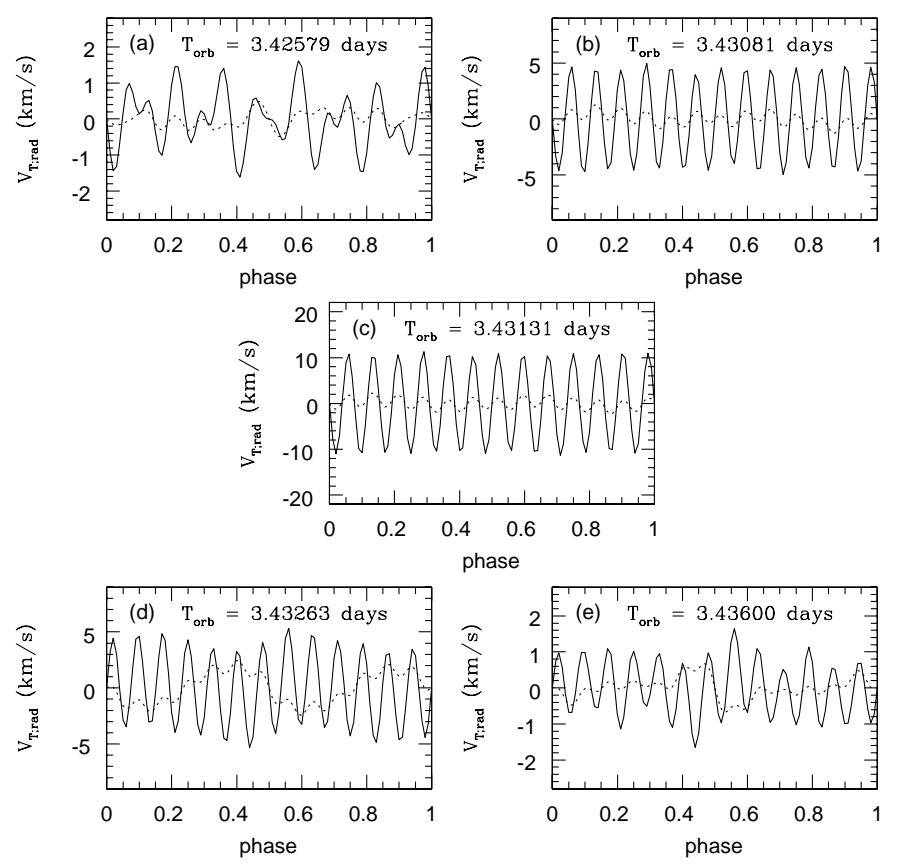

Fig. 6. Observed tidally induced radial-velocity variations of the $5 M_{\odot}$ ZAMS stellar model for orbital periods near 3.43 days, the orbital eccentricity $e=0.5$, and the orbital inclinations $i=20^{\circ}$ (dashed line) and $i=70^{\circ}$ (solid line).

time series and found two intrinsic frequencies for the star: $0.84059 \mathrm{c} . \mathrm{d}^{-1}$ and $0.10108 \mathrm{c} . \mathrm{d}^{-1}$. The first one of these differs less than $0.001{\mathrm{c} . \mathrm{d}^{-1}}^{-1}$ from 10 times the orbital frequency. This observation, together with the binary con- figuration, led De Cat (2001) to conclude that one may be dealing with a resonantly excited mode.

In order to investigate if the suggestion by De Cat (2001) is supported by the theory developed in our paper, we determined the tidal response of a $3.5 M_{\odot}$ stellar model for a fixed orbital period of 11.9154 days as a function of the rotational angular velocity $\Omega$. The model has an age of 64 million years, corresponding to a central hydrogen abundance $X_{\mathrm{c}}=0.6$ and a radius $R_{1}=2.48 R_{\odot}$. The limb-darkening coefficient is assumed to take the value $u=0.36$.

The resulting amplitudes of the tidally induced radialvelocity variations are shown in Fig. 7 for the orbital inclinations $i=90^{\circ}, i=55^{\circ}$, and $i=35^{\circ}$. Since the expressions established in our theory are derived in the linear approximation, we restrict ourselves to the presentation of radial-velocity variations with amplitudes smaller than the sound speed in the atmosphere of the star. The range of rotational angular velocities considered for each inclination is determined from the range of projected equatorial rotation velocities. The companion mass for each inclination results from the mass function of the binary.

A large number of resonances is found for all three orbital inclinations, among which a whole range are caused by partial dynamic tides associated with the Fourier index $k=10$ in Eq. (4) of the tide-generating potential. The azimuthal number of the resonant dynamic tide takes the value $m=-2$ in all these cases, which is compatible with a preliminary mode identification performed by De Cat (2001). While the photometric data did not allow him to identify the mode, he found convincing evidence from the variations of the moments of the line profiles (for a definition see, e.g., Aerts et al. 1992) to conclude that the observed mode is most likely a sectoral $\ell=2$ mode. We note that any resonances caused by partial dynamic tides associated with the azimuthal number $m=0$ would have led to large amplitudes of the tidally induced radial velocity variations for all values of the rotational angular velocity.

In the case of the orbital inclination $i=35^{\circ}$, we indicated the positions of the resonances associated with $m=-2$ and $k=10$ by dotted vertical lines in Fig. 7 . We find a possible resonant excitation of sectoral $\ell=2$ modes ranging from the mode $g_{27}^{+}$up to the mode $g_{53}^{+}$. For the orbital inclinations $i=55^{\circ}$ and $i=90^{\circ}$, the radial orders of the modes excited by the partial dynamic tide associated with $m=-2$ and $k=10$ are even higher.

Upon close inspection, the pattern of resonances in Fig. 7 can be seen to repeat itself at equidistantly spaced values of the rotational angular velocity $\Omega$. The reason is easily identified when the forcing angular frequency $\sigma_{\mathrm{T}}$ is rewritten as

$$
k n+m \Omega=(k+1) n+m\left(\Omega-\frac{n}{m}\right) .
$$

It follows that if the partial dynamic tide associated with the azimuthal number $m$ and the Fourier index $k$ is resonant at the rotational angular velocity $\Omega$, the partial dynamic tide associated with the azimuthal number $m$ and 

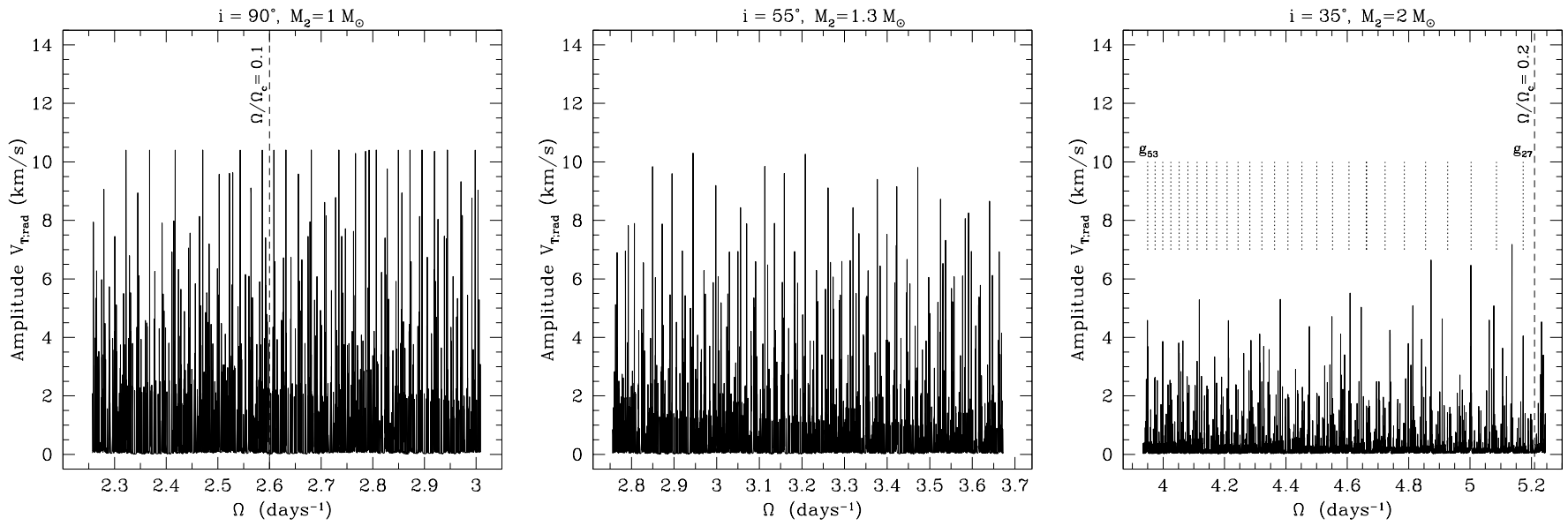

Fig. 7. Observed amplitude of the tidally induced radial-velocity variations in HD 177863 , for the orbital inclinations $i=90^{\circ}$ (left), $i=55^{\circ}$ (middle), and $i=35^{\circ}$ (right). The dashed lines in the left- and right-hand panels indicate the rotational angular velocities corresponding to $10 \%$ and $20 \%$ of the critical break-up angular velocity $\Omega_{\mathrm{c}}$. The dotted lines in the right-hand panel indicate resonances caused by the partial dynamic tide associated with $m=-2$ and $k=10$.

the Fourier index $k+1$ will be resonant at the rotational angular velocity $\Omega-n / m$.

We conclude that due to the large uncertainty in the rotational angular velocity and the orbital inclination of HD 177863, a definitive mode identification is not yet possible at this time. In addition, the amplitude of the tidally induced radial-velocity variations can not be determined in the isentropic approximation so that the inclusion of nonadiabatic effects in our treatment becomes desirable. For higher rotation rates, more resonances can also be expected due to the rotational splitting of the stellar eigenfrequencies by the Coriolis force. We plan to explore the influence of the nonadiabatic effects and the Coriolis force in more detail in subsequent investigations. In particular, we will investigate the behaviour of the predicted observable quantities (photometric variations and line-profile variations) resulting from the candidate resonant modes that we have found in this work with the goal to confront them with the data. Such an iterative procedure can perhaps allow us to come to a definite mode identification and detailed modelling with the goal to constrain the internal structure parameters of the primary.

\section{Concluding remarks}

In this paper, we derived semi-analytical expressions for the tidally induced radial-velocity variations in a uniformly rotating component of a close binary. We neglect the effects of the Coriolis force and the centrifugal force and treat both the free and the forced oscillations of the component as linear, isentropic perturbations of a spherically symmetric star. We take into account the possibility of resonances between dynamic tides and free oscillation modes by means of the perturbation theory developed by Smeyers et al. (1998).

The amplitudes of the tidally induced radial-velocity variations depend on the orbital eccentricity and on the inclination of the orbital plane with respect to the plane perpendicular to the line of sight. They increase with increasing values of the orbital eccentricity and are largest when the orbital plane is seen edge-on. The amplitude is most sensitive to the values of the orbital inclination when $20^{\circ} \lesssim i \lesssim 70^{\circ}$. A similar conclusion was reached by Kumar et al. (1995) in an investigation on the stellar luminosity variations associated with tidally excited oscillation modes.

From the application to a $5 M_{\odot}$ zero-age main sequence star, it follows that the amplitude of the tidally induced radial-velocity variations seen by an observer is small, except when resonances occur between dynamic tides and free oscillations modes. The resonances enhance the tidal motions of the mass elements and lead to radial-velocity variations with amplitudes that are up to an order of magnitude larger than those observed outside resonances. Some orbital periods are seen to give rise to simultaneous resonances with two oscillation modes.

The shape of the tidally induced radial-velocity curves changes markedly with the proximity of a dynamic tide to a resonance with a free oscillation mode. For close resonances, the tidally induced radial-velocity variations are almost exclusively caused by the tide involved in the resonance and therefore exhibit a very sinusoidal-like behaviour. Outside resonance, the radial-velocity variations are less regular functions of the orbital phase.

For conclusion, we applied our results to the slowly pulsating B star HD 177863 and showed the possible resonant excitation of a high-order second-degree sectoral $g^{+}$-mode in this star.

Acknowledgements. The authors express their sincere thanks to Dr. A. Claret for providing them with theoretical stellar models and to an anonymous referee whose valuable comments led to an improvement of the paper. Bart Willems acknowledges the financial support of PPARC grant PPA/G/S/1999/00127. 


\section{Appendix A: The functions $f_{\xi, \ell}(u)$ and $f_{\eta, \ell}(u)$}

The functions $f_{\xi, \ell}(u)$ and $f_{\eta, \ell}(u)$ defined by Eqs. (23) and (24) render the influence of the adopted limbdarkening law on the contributions of the radial and the transverse component of the tidal displacement to the tidally induced radial-velocity variations. In the case of the limb-darkening law given by Eq. (21), the functions $f_{\xi, \ell}(u)$ and $f_{\eta, \ell}(u)$ take the form

$$
\begin{aligned}
f_{\xi, \ell}(u)= & \frac{6}{3-u}\left[(1-u) \gamma_{\ell, 2}+u \gamma_{\ell, 3}\right], \\
f_{\eta, \ell}(u)= & \frac{6}{3-u} \ell\left[(1-u)\left(\gamma_{\ell, 2}-\gamma_{\ell-1,1}\right)\right. \\
& \left.+u\left(\gamma_{\ell, 3}-\gamma_{\ell-1,2}\right)\right],
\end{aligned}
$$

where we have introduced the abbreviation

$\gamma_{\ell, n} \equiv \int_{0}^{1} P_{\ell}(x) x^{n} \mathrm{~d} x$.

For non-negative values of $n$, the integral in the right-hand member of Eq. (A.3) yields

$\gamma_{\ell, n}=\frac{\pi^{1 / 2} \Gamma(n+1)}{2^{n+1} \Gamma[(n-\ell+2) / 2] \Gamma[(n+\ell+3) / 2]}$,

(Abramowitz \& Stegun 1965, Eq. (8.14.15)).

In the particular case where $\ell=2$, the functions $f_{\xi, 2}(u)$ and $f_{\eta, 2}(u)$ take the form

$f_{\xi, 2}(u)=\frac{1}{20} \frac{16-u}{3-u}$,

$f_{\eta, 2}(u)=-\frac{3}{10} \frac{8-3 u}{3-u}$

\section{References}

Abramowitz, M., \& Stegun, I. A. 1965, Handbook of mathematical functions with formulas, graphs, and mathematical tables (Dover Books on Advanced Mathematics, New York)
Aerts, C., De Mey, C., De Cat, P., \& Waelkens, C. 1998, in A Half Century of Stellar Pulsation Interpretation: A Tribute to Arthur N. Cox, ed. P. A. Bradley, \& J. A. Guzik, ASP Conf. Ser., 135, 380

Aerts, C., de Pauw, M., \& Waelkens, C. 1992, A\&A, 266, 294 Chapellier, E., Le Contel, J. M., Le Contel, D., Sareyan, J. P., \& Valtier, J. C. 1995, A\&A, 304, 406

Clausen, J. V. 1996, A\&A, 308, 151

Cowling, T. G. 1941, MNRAS, 101, 367

De Cat, P. 2001, An observational study of bright southern slowly pulsating B stars, Ph.D. Thesis, Katholieke Universiteit Leuven, Belgium

De Cat, P., Aerts, C., De Ridder, J., et al. 2000, A\&A, 355, 1015

Eggen, O. J. 1977, PASP, 89, 187

Fitch, W. S. 1967, ApJ, 148, 481

Fitch, W. S., \& Wisniewski, W. Z. 1979, ApJ, 231, 808

Green, R. M. 1985, Spherical astronomy (Cambridge university press, Cambridge)

Harmanec, P., Hadrava, P., Yang, S., et al. 1997, A\&A, 319, 867

Jeffreys, B. 1965, Geophys. J., 10, 141

Kumar, P., Ao, C. O., \& Quataert, E. J. 1995, ApJ, 449, 294

Polfliet, R., \& Smeyers, P. 1990, A\&A, 237, 110

Rocca, A. 1987, A\&A, 175, 81

Smeyers, P., Willems, B., \& Van Hoolst, T. 1998, A\&A, 335, 622

Smith, M. A. 1985a, ApJ, 297, 206

Smith, M. A. 1985b, ApJ, 297, 224

Waelkens, C. 1991, A\&A, 246, 453

Waelkens, C., \& Rufener, F. 1985, A\&A, 152, 6

Westin, T. N. G. 1985, A\&AS, 60, 99

Willems, B. 2000, Contribution to the linear theory of dynamic tides and orbital evolution in close binaries, Ph.D. Thesis, Katholieke Universiteit Leuven, Belgium

Willems, B., Smeyers, P., \& Van Hoolst, T. 2001, A\&A, submitted

Willems, B., Van Hoolst, T., Smeyers, P., \& Waelkens, C. 1997, A\&A, 326, L37

Witte, M. G., \& Savonije, G. J. 1999, A\&A, 350, 129

Witte, M. G., \& Savonije, G. J. 2001, A\&A, 366, 840

Zahn, J. P. 1970, A\&A, 4, 452 